\title{
An Effective Modeling for Face Recognition System: LDA and GMM based Approach
}

\author{
Aditi Mandloi \\ Shri Vaishnav Institute of Technology and Science \\ Indore, India
}

\begin{abstract}
A broad selection of systems need reliable personal recognition schemes to either verify or decide the identity of an entity requesting their services. The cause of such schemes is to make sure that the rendered services are accessed only by a genuine user, and nobody else. It is widely acknowledged that the face identification has played a significant role in the observation system as it doesn't need the object's assistance. The definite advantages of face based recognition over other biometrics are distinctiveness and response. As human face is an active object having a high degree of unpredictability in its manifestation, that makes face detection a hard problem in computer vision. In this work we presented a novel Face Recognition feature Extraction Mode based on the combination of Linear Discriminant Analysis (LDA) and Gaussian Mixture Model (GMM). The proposed LDA and GMM based feature Extraction Model is utilized to search the feature space for the top feature subset where features are carefully selected according to a well-defined discrimination criterion. For the betterment of the feature classification a KNN classifier is used. The classifier performance and the length of choosing a feature vector measure for performance estimation using MATLAB in ORL face dataset.
\end{abstract}

\section{Keywords}

Face Recognition, Feature selection, LDA, ORL Dataset, Authentication, Gaussian Mixture Model;

\section{INTRODUCTION}

The human face plays a crucial role in our social interaction, transfer people's identity. Exploitation the human face as a key to security, biometric face recognition technology has received important attention in the past many years as a result of its potential for a good type of applications in each law enforcement and non-law enforcement. A good type of systems need reliable personal recognition schemes to either make sure or verify the identity of a person requesting their services. The aim of such schemes is to confirm that the rendered services are accessed solely by the legitimate user and not any one else.

\subsection{Face Recognition}

It is the overall opinion that advances in computer vision analysis can give helpful insights to neuroscientists and psychologists into however human brain works, and vice versa. Face recognition is one of the foremost relevant applications of image analysis. It's a real challenge to make an automatic system which equals human ability to acknowledge faces. Although humans are quite sensible identifying known faces, we tend to be not very skilled after we should take care of a large quantity of unknown faces. The computers, with a virtually limitless memory and computational speed, should overcome human's limitations. The face recognition techniques primarily add three steps [2].

\author{
Priyanka Gupta \\ Shri Vaishnav Institute of Technology and Science \\ Indore, India
}

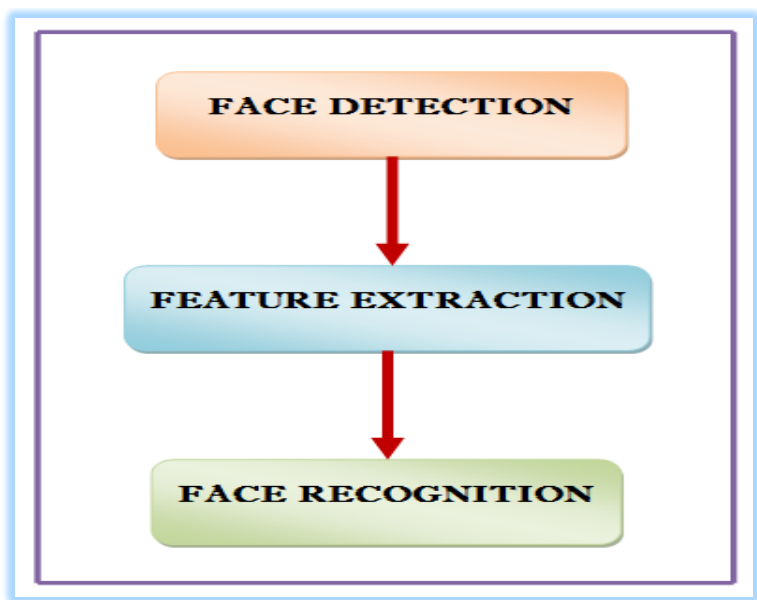

Fig. 1: Face detection and Recognition Overview
a. Face Detection,
b. Feature Extraction,
c. Face Recognition.

a. Face Detection

Face detection is regarded as a particular case of object-class detection. In object-class detection, the task is to find the locations and sizes of all objects in an image that belong to a given class. Face detection is regarded as a lot of general cases of face localization. In face localization, the task is to search out the locations and sizes of a known number of faces (usually one). In face detection, one doesn't have this additional information.

b. Feature Extraction

The In pattern recognition and in image process, feature extraction could be a special variety of dimensionality reduction. Once the input data to an algorithm is simply too large to be processed and it's suspected to be notoriously redundant then the input data will be remodeled into a reduced representation set of features. After the FD step, human-face patches are extracted from images. Directly using these patches for FR has some disadvantages, first, every patch usually contains over a thousand pixels that are overlarge to make a strong recognition system. Second, face patches may be taken from totally different camera alignments, with totally different facial expressions, illuminations, and will suffer from occlusion and muddle. To overcome these drawbacks, feature extractions are performed to try and do info, packing, dimension reduction, salience extraction, and noise improvement. Once this step, a face patch is typically remodeled into a vector with fastened dimension or a set of fiduciary points and their corresponding locations. Remodeling the input data into the set of options is called feature extraction [3]. 


\section{c. Face Recognition}

An automatic face recognition system is a computer application for automatically identifying or verifying someone from a digital image or a video frame from a video source. One in all the ways that try to do this is often by comparing selected facial features from the image and a facial database. It usually used in security systems and can be compared to different biometrics like fingerprint or eye iris recognition systems. Among the various biometric techniques, automatic face recognition might not be the foremost reliable and efficient. However, one key advantage is that it doesn't require aid (or consent) from the test subject. Properly designed systems installed in airports, multiplexes, and different public places will determine people among the group.

\section{A. LDA}

LDA is wide used to realize linear combinations of features whereas preserving class separability. Unlike PCA, LDA tries to model the variations between classes. Classic LDA is meant to require into account solely 2 classes. Specifically, it needs knowledge points for different classes being far from one another, whereas points from an equivalent category are shut. Consequently, LDA obtains difference projection vectors for each class. Multi-class LDA algorithms which may manage more than 2 classes are a lot of used. Linear Discriminant Analysis (LDA) is most commonly used as a dimensionality reduction technique within the preprocessing step for patternclassification and machine learning applications. The goal is to project a data set onto a lower-dimensional space with good class-separability so as avoid over fitting ("curse of dimensionality") and also reduce machine costs [4].

\section{B. Gaussian Mixture Model}

A Gaussian mixture model (GMM) is beneficial for modeling data that comes from one of several teams: the groups might be totally different from one another, but data points within a similar cluster are well-modeled by a distribution. GMM provides a mathematical approach to applied math modeling of a large form of random phenomena and also provides a semi parametric framework to model an unknown likelihood density distribution shape. The mixture of GMM with linear projection schemes is becoming popular because it provides quite one transformation matrix. Gaussian Mixture Models is accustomed represent probability density functions complicated, from the marginalization of joint distribution between discovering variables and hidden variables. Gaussian mixture model relies on the fact that a big number of probability distributions can be approximated by a weighted total of Gaussian functions [5], [6].

A Gaussian mixture model is a weighted sum of $\mathrm{k}$ component Gaussian densities for vector $\mathrm{x}$ as given by the equation,

$$
p(x)=\sum_{j=1}^{k} \alpha_{j} p(x / j)
$$

Where $\mathrm{x}$ is a D-dimensional continuous-valued data vector (i.e. Measurement or features)

$\alpha_{\mathrm{j}}=1,2, \ldots \mathrm{k}$, are the Mixture Weights

$p(x / j)=$ Gaussian Density Model for $j^{\text {Th }}$ Component,

Each component density is a D-variate Gaussian function of the form,

$$
p(x / j)=\frac{1}{(2 \pi)^{1 / d}} e^{-1 / 2\left(x-\mu_{j}\right)^{T} \sum j^{-1}\left(x-\mu_{j}\right)}
$$

With a mean vector $\mu_{j}$ And covariance matrix $\sum j$. The mixture weights satisfy the constraint that $\sum_{i=1}^{k} \alpha_{j}=1$.

Model parameter estimation is performed using a well known iterative method called Expectation Maximization (EM) which assumes that the number of components is known beforehand.

The complete Gaussian mixture model is parameterized by the mean vectors, covariance matrices and mixture weights from all component densities. These parameters are collectively represented by the notation $\mu_{j}$ And $\sum j$

\section{LITERATURE SURVEY}

Through the past three decades in the face recognition systems have been developed. Indeed, this development depending on three kinds of recognition algorithms, namely profile, frontal, and view tolerant recognition, based on the type of images and the recognition algorithms.

The Gaussian mixture model (GMM) supervector approach is a well known technique in the domain of speech processing, e.g. speaker verification and audio segmentation. In this paper Martin Schulz et al. [7] Apply this approach to video data in order to recognize human facial expressions. Three different image feature types (optical flow histograms, orientation histograms and principal components) from four pre-selected regions of the human face image were extracted and GMM supervector of the feature channels per sequence were constructed. Support vector machines (SVM) were trained using these supervector for every channel separately and its results were combined using classifier fusion techniques. Thus, the performance of the classifier could be improved compared to the best individual classifier.

Wen Wang et al. [8] Presents a method named Discriminant Analysis on Riemannian manifold of Gaussian distributions (DARG) to solve the problem of face recognition with image sets. Our goal is to capture the underlying data distribution in each set and thus facilitate more robust classification. To this end, we represent image set as a Gaussian Mixture Model (GMM) comprising a number of Gaussian components with prior probabilities and seek to discriminate Gaussian components from different classes. In the light of information geometry, the Gaussians lie on a specific Riemannian manifold. To encode such Riemannian geometry properly, we investigate several distances between Gaussian and further derive a series of provably positive definite probabilistic kernels. Through these kernels, a weighted Kernel Discriminant Analysis is finally devised which treats the Gaussians in GMMs as samples and their prior probabilities as sample weights. The proposed method is evaluated by face identification and verification tasks on four most challenging and largest databases, YouTube Celebrities, COX, YouTube Face DB and Point-and-Shoot Challenge, to demonstrate its superiority over the state-of-the-art.

In this paper, Najim DEHAK et al. [9] Present a new face recognition algorithm. It supposes that a video sequence of a person is available both in enrollment and test time. During enrollment, a client Gaussian Mixture Model (GMM) is adapted from a world GMM using eigenface features extracted from each frame of the video. Then, a Support Vector Machine (SVM) is used to find a decision border between the client GMM and pseudoimpostors GMMs. At test time, a GMM is adapted from the test video and a decision is taken using the previously learned client SVM. This algorithm brings a 3.5\% Equal Error Rate (EER) improvement over the BioSecure reference system on the Pooled protocol of the BANCA database. 
Low-dimensional feature representation with enhanced discriminatory power is of paramount importance to face recognition (FR) systems. Most of the traditional linear discriminant analysis (LDA) -based methods suffer from the disadvantage that their optimality criteria are not directly related to the classification ability of the obtained feature representation. Moreover, their classification accuracy is affected by the "small sample size" (SSS) problem which is often encountered in FR tasks. In this short paper, Kostantinos $N$, et al. [10] Propose a new algorithm that deals with both of the shortcomings in an efficient and cost effective manner. The proposed here method is compared, in terms of classification accuracy, two other commonly used FR methods on two face databases. Results indicate that the performance of the proposed method is overall superior to those of traditional FR approaches, such as the Eigenfaces, Fisherfaces, and D-LDA methods.

With the goal of matching unknown faces against a gallery of known people, the face identification task has been studied for several decades. There are very accurate techniques to perform face identification in controlled environments, particularly when large numbers of samples are available for each face. However, face identification under uncontrolled environments or with a lack of training data is still an unsolved problem. William Robson Schwartz et al. [11] employ a large and rich set of feature descriptors (with more than 70000 descriptors) for face identification using partial least squares to perform multichannel feature weighting. Then, we extend the method to a tree-based discriminative structure to reduce the time required to evaluate probe samples. The method is evaluated on Facial Recognition Technology (FERET) and Face Recognition Grand Challenge (FRGC) data sets. Experiments show that our identification method outperforms current state-of-the-art results, particularly for identifying faces acquired across varying conditions.

\section{PROPOSED WORK}

The proposed system is designed in two different data sets in which one data set passes to process training to the LDA and GMM and the other is passed for testing on LDA and GMM. Here in the training module system accepts user data in form of Image and calculate features. In testing phase system accepts user ORL data set in the form of image and the system produces features. On the basis of these calculated features we classify features by using K-nearest neighbor algorithm. The basic overview of the system is given below.

\subsection{Methodology}

The working of the proposed algorithm is given and explained in details in this section. To understand the core concept of the proposed methodology by using given figure 2 descriptions:

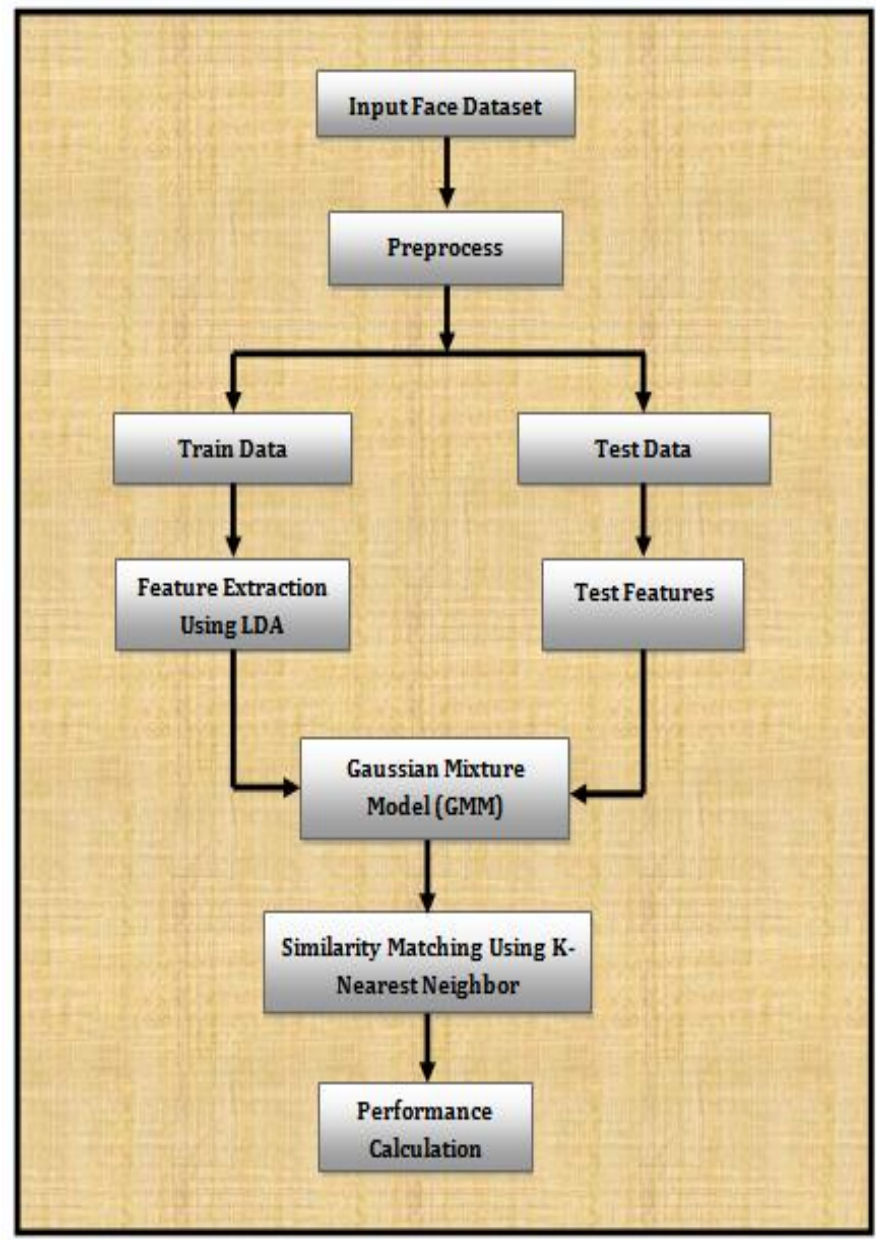

Fig. 2: Proposed Working Flow

* Input face dataset: To process the entire project we need to load the face dataset. In this we give the input as ORL data set. While loading the input data set that process for the next step and initialize the process.

* Preprocess: This is done to enhance the images to improve the recognition performance of the system. After this process, our data set split into two different datasets, i.e. train data set and test data set. Different function performs on this dataset.

* Train Data: This is the data on which we performed training to train the model. On the basis of this model we process further steps

* Test Data: Similarly, this is the data on which we performed testing process to test the model. For this testing does measure for accuracy of the entire system

- Feature Extraction using LDA: This module finds the key features in the face that will be used in classification. It is responsible for composing a feature vector that is well enough to represent the faces. For this purpose we apply the standard LDA algorithm for extracting the image features.

* Test Feature: This is a test feature which is calculated from the test dataset.

* Gaussian Mixture Model: On this model, we give input feature which is calculated by LDA along with give test features to process the function. GMM used for modeling the shape and texture of face images. 
* Similarity matching using KNN: By modeling of GMM, we get optimal extracted features of this input image. Likewise, NN classify the different classes of similar attribute basis. NN classify the image faces of different 40 classes. That class contains 400 face images, i.e., each class contains 10 face images.

* Performance Calculation: Finally on the basis of the proposed working flow we can conclude that system using efficient performance of the face recognition basis of the following parameter 1) Recognition Rate, 2) Verification Rate, 3) Error rate.

\subsection{Proposed Algorithm}

The given section provides the detailed understanding about the proposed algorithm face recognition system:

\section{Table 1: Proposed Algorithms}

Input: ORL face Dataset,

Output: Performance Parameter for classification Accuracy

\section{Process:}

1: Load input face dataset (ORL)

2: Bifurcate data into train dataset and test data set

3: Feature Extraction using LDA method. Following are the LDA steps:

- $\quad$ Suppose there are $\mathrm{C}$ classes

- $\quad$ Let $\mu_{i}$ be the mean vector for class $i, i=1,2,3,4, \ldots C$

- Let $M_{i}$ be the number of samples within class $i, i=$ $1,2,3,4, \ldots C$

- $\quad$ Let $M=\sum_{i=0}^{C} M_{i}$ Be the total number of samples, and

Within Class Scatter Matrix : $S_{w}=\sum_{i=1}^{C} \sum_{j=1}^{M_{i}}\left(y_{j}-\right.$ $\mu i) y j-\mu i T$

Between Class Scatter Matrix: $S_{b}=\sum_{i=1}^{C} \sum_{j=1}^{M_{i}}\left(\mu_{i}-\right.$ $\mu) \mu i^{-} \mu T$

$\mu=\frac{1}{C} \sum_{i=1}^{C} \mu_{i}$ (mean of entire dataset)

- LDA computes a transformation that maximizes the between-class scatter while minimizing the within-class scatter:

$$
\operatorname{maximize} \frac{\operatorname{det}\left(S_{b}\right)}{\operatorname{det}\left(S_{w}\right)}
$$

- Such a transformation should retain class separability while reducing the variation due to sources other than identity (e.g., illumination).

4: Calculate test feature of the test data set

5: Apply GMM on training and testing features. Following are the GMM steps:

Probability density function (PDF) with $k$ component for feature vector $x$ is

- $\quad p(x)=\sum_{j=1}^{k} \alpha_{j} p(x / j) / / \boldsymbol{\alpha}_{j}=$ Mixture Weight,

$/ / p(x / j)=$ Gaussian Density Model for $j^{\text {th }}$ Component
- $p(x / j)=\frac{1}{(2 \pi)^{1 / d}} e^{-1 / 2\left(x-\mu_{j}\right)^{T} \sum j^{-1}\left(x-\mu_{j}\right)} / / \mu_{j}=$ Mean

Vector,

\section{$/ / \sum j=$ Covariance Matrix for $j^{\text {th }}$ Component}

- $\quad \mu_{j}$ And $\sum j$ Have to be calculated using Expectation Minimization Function

- $\quad p\left(j / x_{i}\right)=\frac{\alpha_{j} p\left(x_{i} / j\right)}{\sum_{i=1}^{k} \alpha_{i} p\left(x_{i} / t\right)}$

- $\quad \alpha_{j}=\frac{\sum_{i=1}^{n} p\left(j / x_{i}\right)}{n}$

- $\quad \mu_{j}=\frac{\sum_{i=1}^{n} p\left(j / x_{i}\right) x_{i}}{\sum_{i=1}^{n} p\left(j / x_{i}\right)}$

- $\quad \sum j=\frac{\sum_{i=1}^{n} p\left(j / x_{i}\right)\left(x_{i}-\mu_{j}\right)\left(\left(x_{i}-\mu_{j}\right)^{T}\right.}{\sum_{i=1}^{n} p\left(j / x_{i}\right)}$

6: Perform Similarity matching using K-nearest neighbor

7: Compute System Performance

8: Return Classification Accuracy

9: End Process

\section{RESULT ANALYSIS}

\subsection{Verification Rate (ROC curve)}

Verification rate relies on a list of image pairs, where pair with the same and pairs with different identities are compared

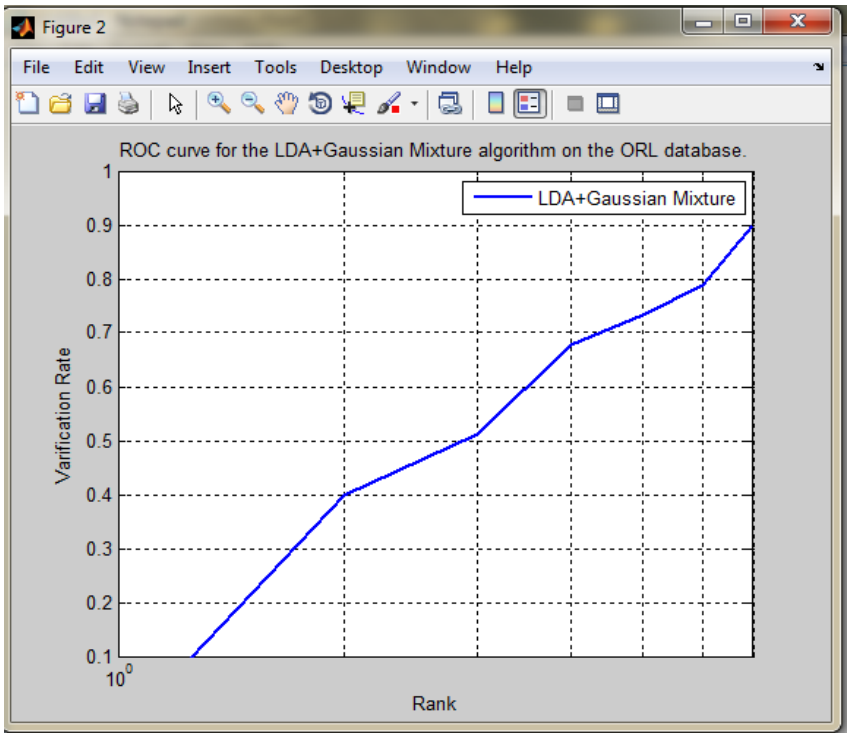

Fig. 3: Verification Rate

The ROC curve for the proposed system has given the figure 3 shows the ROC for face recognition using LDA and GMM based method. In order to represent the performance of the system $\mathrm{X}$ axis shows the rank of the data and the $\mathrm{Y}$ axis contains the verification rate of the input patterns. According to the obtained performance of system the face recognition rate for the classification is higher as other traditional classifiers.

\subsection{Recognition Rate (CMC Curve)}

The recognition rate is how many images correctly matching with the training images, while false acceptance is how many images (outside the dataset) are matched with the dataset images. 


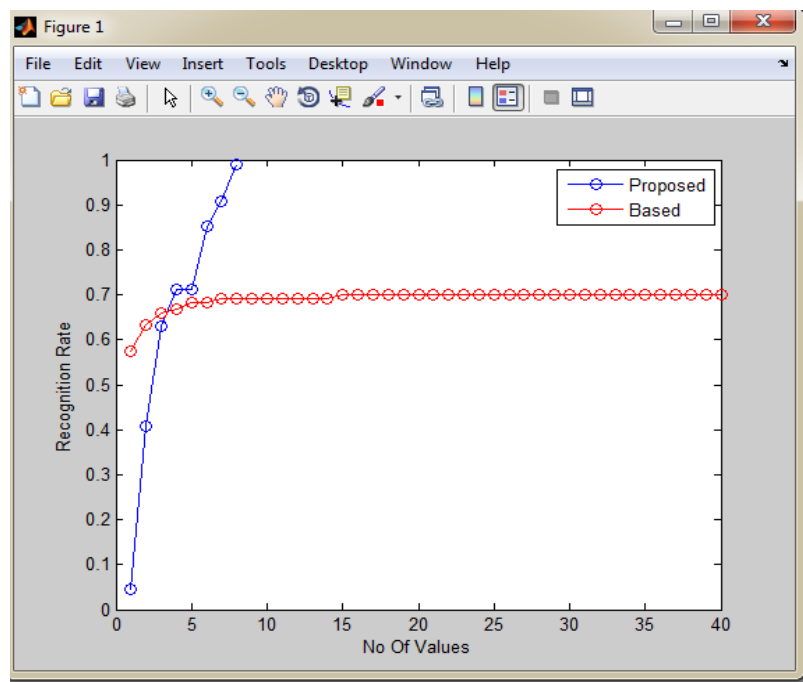

Fig. 4: Recognition Rate

In above figure 4 depict the performance of LDA and GMM for face recognition. According to the obtained results the accuracy of the proposed, implemented systems is adoptable. The result identified by the plotting values of the graph. In this $\mathrm{X}$-axis show the rank of the plotting values and $\mathrm{Y}$-axis represent the produced verification rate. The result uses ORL data set for evaluating feature from the faces. Nevertheless, pattern matching is satisfied by the single coherent extracting data of the processing of LDA. After that GMM is calculating receive features. Hence the given output of the proposed system is very efficient of a combination of LDA and GMM.

\subsection{Error Rate (EPC Curves)}

An error rate of face recognition can be defined as the faces for the class which are not matched correctly to the available faces. Those faces are not matched that show the percentage of the error rate.

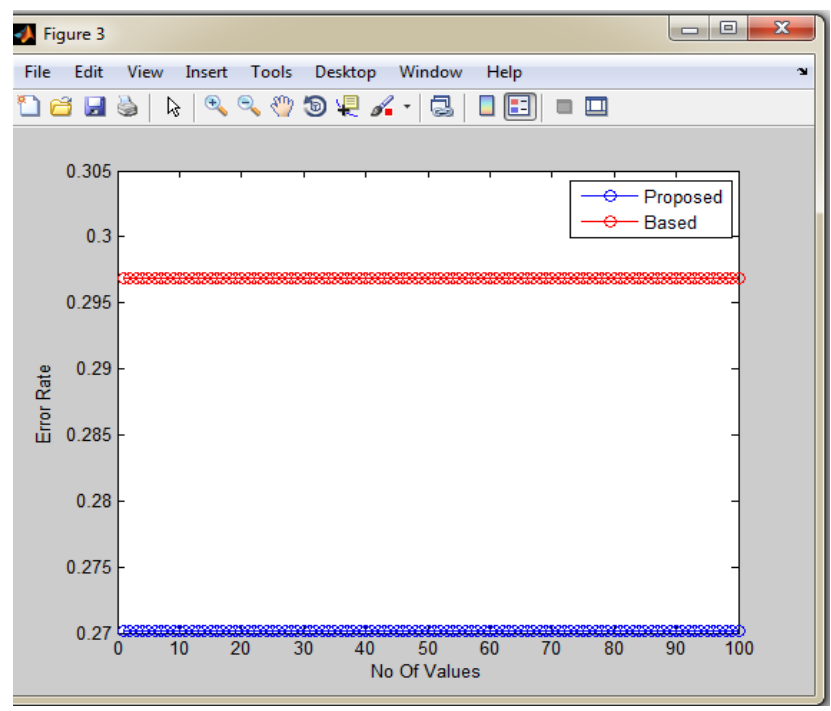

Fig. 5: Error Rate

The performance of the system in terms of LDA and GMM based face recognition is given using figure 5. That actually represents the error rate of the system if the error rate of the classification is less than the performance is improving and providing much accurate classification rate. Thus the proposed model for LDA+GMM Face recognition is adaptable with the lowest error rate during the recognition task.

\section{CONCLUSION}

Facial recognition technologies have generally been associated with very costly top secure applications. Today the core technologies have evolved and the cost of equipments is going down dramatically due to the integration and the increasing processing power. Certain applications of face recognition technology are now cost effective, reliable and highly accurate. As a result, there are no technological or financial barriers for stepping from the pilot project to widespread deployment. Though there are some weaknesses of facial recognition systems, there is a tremendous scope in India. This system can be effectively used in $\mathrm{ATM}^{\text {ee }} \mathrm{s}$, identifying duplicate voters, passport and visa verification, driving license verification, in defense, competition and other exams, in governments and private sectors. The Experimental results showed that the proposed algorithm performs better with the face recognition using multi model image features and produce effective classification result with the help of KNN classifier. Optimal Feature Extraction (FE) is to find the minimal subset of features from the original feature set that can represent the whole data set. In this paper, a novel LDA and GMM based Face Recognition algorithm are proposed. In this domain the training and testing are the two phases for pattern learn from the past experience. These patterns are used by the computational model to prepare a data model by which the target objectives are accomplished. These techniques are frequently used in the new generation for optimizing the experience of human life. Thus the GMM is suitable for applications when large databases must be handed and they change with the time, as in real time face recognition operations.

\section{FUTURE WORK}

There are several directions where this work can be extended. Concept of Soft Computing can be used for the automatic face recognition system.

$\checkmark$ Using Soft Computing, neural network can be combined with fuzzy logic to enhance the performance of face recognition.

$\checkmark$ Another avenue for research would be to implement other feature extraction techniques on the same data set.

$\checkmark \quad$ In the future, two or more classifiers can be combined to achieve better results.

\section{REFERENCES}

[1] Rein-Lien Hsu, "Face Detection and Modeling for Recognition," PhD thesis, Department of Computer Science \& Engineering, Michigan State University, USA, 2002

[2] B. Maison,C. Neti, and A. Senior, Audio-visual Speaker Recognition for Video Broadcast News: Some Fusion Techniques, In Multimedia Signal Processing, 1999.

[3] Wilson, Phillip Ian, and John Fernandez, "Facial feature detection using Haar classifiers", Journal of Computing Sciences in Colleges 21.4 (2006): 127-133.

[4] Sebastian Raschka, "Linear Discriminant Analysis", available online at: http://sebastianraschka.com/Articles/2014_python_lda.html \#summarizing-the-lda-approach-in-5-steps

[5] Mitra, Sinjini. "Gaussian mixture models for human face recognition under illumination variations." (2012).

[6] Hassanpour, Reza, Asadollah Shahbahrami, and Stephan Wong, "Adaptive Gaussian mixture model for skin color 
segmentation", World Academy of Science, Engineering and Technology 41 (2008): 1-6.

[7] Wang, Wen, et al, "Discriminant analysis on Riemannian manifold of Gaussian distributions for face recognition with image sets", Proceedings of the IEEE Conference on Computer Vision and Pattern Recognition. 2015.

[8] Schels, Martin, and Friedhelm Schwenker, "A multiple classifier system approach for facial expressions in image sequences utilizing GMM supervectors", Pattern Recognition (ICPR), 2010 20th International Conference on, IEEE, 2010.
[9] Bredin, Hervé, Najim Dehak, and Gérard Chollet, "GMMbased SVM for face recognition", Pattern Recognition, ICPR 2006, 18th International Conference on, Vol. 3, IEEE, 2006.

[10] Lu, Juwei, Konstantinos N. Plataniotis, and Anastasios N Venetsanopoulos, "Face recognition using LDA-based algorithms", IEEE transactions on neural networks 14.1 (2003): pp. 195-200.

[11] Schwartz, William Robson, et al. "Face identification using large feature sets." IEEE Transactions on Image Processing 21.4 (2012): pp. 2245-2255. 